\section{Fatigue at work}

\section{F J H van Dijk, G M H Swaen}

\section{Understanding how acute and chronic fatigue have an adverse impact on the health of workers}

$\mathrm{F}$ atigue is a common complaint in the working population. Approximately $20 \%$ of the working population report symptoms that fall under the concept of fatigue. ${ }^{1}$ Other surveys have reported prevalence rates of fatigue varying from $7 \%$ to $45 \%$, depending on the instruments used and the applied cut off points. ${ }^{2}$

At first sight, the presence of fatigue in a working population does not give much reason for concern from an occupational health perspective. After work has been done, some fatigue may be expected and this fatigue is compensated for by social and financial rewards. Occupational health interest in fatigue arises from the adverse consequences that are attributed to fatigue in the more serious acute or chronic forms, and when there is insufficient opportunity to for workers to recover. Bad performance, impaired quality of services and products, and dropout of personnel may be adverse consequences. For employees, feelings of professional incompetence, accidents, and fatigue's disruptive effects on private social life are serious outcomes. An increased risk of depression, as well as infectious and cardiovascular diseases have been suggested. Effects may be so serious that workers can experience disability leading to long term or even indefinite absenteeism from work. Stress related disorders are labelled under quite a number of interrelated terms and definitions such as adjustment disorders, neurasthenia, nervous breakdown, surmenage, and burnout. Prolonged fatigue is one of the core symptoms, and stress related disorders are responsible for approximately $30 \%$ of permanent disability benefits in the Netherlands. In other countries, such as the UK, mental ill health is a growing concern as it is causing increased absenteeism and long term sickness. ${ }^{3}$

The Dutch Organisation for Scientific Research (NWO) recognised the substantial public health significance of this topic and in 1996 funded the Priority Research Programme titled "Fatigue at Work". Within this programme Dutch universities, various other institutes, and (occupational) health care institutions participated in multidisciplinary projects to acquire a better understanding of fatigue's causes and consequences. Projects were included on acute fatigue during the working day as well as projects on burnout and on work related adjustment disorders. All projects aimed at improving our understanding of the aetiology, course, and consequences for human functioning in acute and prolonged states of fatigue. A number of projects were oriented to concepts, aetiology, and prognosis, others were oriented to surveillance, diagnosis, therapy, or rehabilitation of workers after a period of sickness absence.

The Priority Research Programme, chaired by Professor T Meijman, was planned as a multidisciplinary initiative. At the start of the programme fatigue was defined as "the change in the psychophysiological control mechanism that regulates task behaviour, resulting from preceding mental and/or physical efforts which have become burdensome to such an extent that the individual is no longer able to adequately meet the demands that the job requires of his or her mental functioning; or that the individual is able to meet these demands only at the cost of increasing mental effort and the surmounting of mental resistance." This definition principally implies that fatigue in itself is not an adverse effect but rather a physiological adaptation or safety mechanism of the individual confronted with the risk of overstrain or exhaustion. Physiological fatigue can be seen as a feedback mechanism that will reduce drive and motivation in instances where exhaustion can lead to adverse mental or physical effects. Finally, in quite a number of mental disorders including burnout, adjustment disorders (DSM IV and ICD$10)$, and neurasthenia (ICD-10), prolonged or chronic fatigue is one of the core symptoms. The presence of many other related terms and definitions such as nervous exhaustion, nervous breakdown, and surmenage refers to the confusion related to the concepts of a number of psychological syndromes and minor psychiatric disorders, which are closely related to fatigue. The wide range of frequently used terms and concepts also reflects the relevance of the topic. Most extensively used scales to measure fatigue or related entities within the research programme are the Checklist Individual Strength (CIS), the Need for Recovery scale (van Veldhoven and Broersen, p i3), ${ }^{4}$ and the Maslach Burnout Inventory (MBI). In a recent paper by Shapiro and Moller instruments are described including the CIS. ${ }^{5}$

It is clear that many questions are unanswered like the relationships between working conditions and fatigue, acute fatigue and prolonged or chronic fatigue, or fatigue and adverse consequences. The concepts mentioned above are confusing and thus in cases of serious problems diagnostics is an important topic. Consequently, the effectiveness of treatment and rehabilitation in disorders where fatigue is a core symptom are unknown. These and other questions were the reason to start this research programme.

In this supplement we do not present a complete overview of the projects of the programme, nor do the articles represent all research areas. Instead we present interesting results of a programme in progress. They all share the perspective of aspects of epidemiology or care. A number of projects originate from one comprehensive follow up study. The same concepts and instruments have been applied in many projects but there are also differences in perspectives and instruments. Several articles in this supplement address the concept and measurement of fatigue and related problems and disorders. De Vries et al ( $p$ i10) compare the psychometric qualities of six fatigue questionnaires in a working population in an effort to assess validity and to answer the question of mono or multidimensionality of fatigue in a working population. ${ }^{6}$ van Veldhoven and Broersen ( $\mathrm{p}$ i3) introduce the Need for Recovery scale as a valid instrument to assess fatigue. ${ }^{4}$ Brenninkmeijer and Van Yperen ( $p$ il6) discuss the mono or multidimensional structure of the Maslach Burnout Inventory and the use of a cut off point in a non-clinical population. ${ }^{7}$ Huibers et al (p i26) concentrate on the overlap and differences between employees on sick leave with severe fatigue and chronic fatigue syndrome who are under treatment with health care practitioners. ${ }^{8}$ In other contributions issues of diagnosis are addressed. Nieuwenhuijsen et al ( $p$ i21) report about the validity and feasibility of the Depression Anxiety Stress Scales to differentiate in occupational health care between employees with stress related disorders and employees with more serious psychiatric disorders. ${ }^{9}$ Fatigue attributions that employees make during fatigue related visits to general physicians are analysed by Andrea and coworkers (p i99), searching for questions informing health care practitioners about underlying health problems. ${ }^{10}$ De Vente et al (p i54) focus on differences in 
physiological values and stress responses between burnout patients and healthy controls. ${ }^{11}$

The epidemiological approach has been conceptualised in a large prospective follow up study introduced by Kant et al ( $\mathrm{p}$ i32) describing the study model and design. ${ }^{12}$ In this contribution conceptual associations of prolonged fatigue are presented as well as their prevalences including the differences between sectors, companies, and departments. Jansen et al (p i47) studies the influence of work schedules on fatigue as well as the impact of fatigue on withdrawal from shift work. ${ }^{13}$ Acute stressors in ambulance work could be related to chronic fatigue, which has been studied by van der Ploeg and Kleber (p i40).${ }^{14}$ The influence of other working conditions was taken into consideration. Weijman et al (p i93) analysed a large sample of employees with diabetes mellitus searching for disease and work related determinants of fatigue, and potential interactions. ${ }^{15}$ In a paper by Sluiter et al (p i62) results are presented of six studies in which working conditions were studied as causes of fatigue in six different occupations. ${ }^{16}$ Subjective health complaints and sickness absence are analysed as potential consequences. Sickness leave was also the object of study by Janssen et al (p i71) answering the question if fatigue is an independent predictor of sickness absence. ${ }^{17}$ van Amelsvoort et al ( $\mathrm{p}$ i83) inform us about the relative risk of cardiovascular diseases under the condition of fatigue, analysed in the large prospective follow up study mentioned before. ${ }^{18}$ In a study by Swaen et al (p i88) the risk for being injured in an occupational accident was the object of study. ${ }^{19}$ Finally, Nieuwenhuijsen et al ( $\mathrm{p}$ i77) relate quality of rehabilitation to sickness absence duration in cases of nervous breakdown, using performance indicators. ${ }^{20}$ The aim is to improve quality of care.

This supplement presents a multidisciplinary approach to a topic that may turn out to be one of the most relevant research issues in occupational health in the future.

Occup Environ Med 2003;60(Suppl I):i 1-i2

\section{Authors' affiliations}

F J H van Dijk, Coronel Institute for Occupational and Environmental Health, Academic Medical Center, AmCOGG, University of Amsterdam, The Netherlands G M H Swaen , Department of Epidemiology Maastricht, University Maastricht, The Netherlands

\section{REFERENCES}

1 Bültmann U, Kant I, Kasl SV, et al. Fatigue and psychological distress in the working population psychometrics, prevalence, and correlates. J Psychosom Res 2002;52:445-52

2 Lewis G, Wessely S. The epidemiology of fatigue: more questions than answers. J Epid Community Health 1992:46:92-97.

3 Glozier N. Mental ill health and fitness for work. Occup Environ Med 2002;59:714-20.

4 van Veldhoven $M$, Broersen S. Measurement quality and validity of the Need for Recovery Scale. Occup Environ Med 2003:60:i3-9.

5 Shapiro CM, Moller HJ. Chronic fatigue: listen and measure. J Psychosom Res 2002;52:427-36.

6 De Vries J, Michielsen HJ, Van Heck GL. Assessment of fatigue among working people: a comparison of six questionnaires. Occup Environ Med 2003;60:i 10-5.

7 Brenninkmeijer V, Van Yperen N. How to conduct research on burnout: about the advantages and disadvantages of a unidimensional approach in burnout research. Occup Environ Med 2003;60:i 16-20.

8 Huibers MJH, Beurskens AJHM, Prins JB, et al. Fatigue, burnout, and chronic fatigue syndrome among employees on sick leave: do attributions make the difference? Occup Environ Med 2003;60:i26-31

9 Nieuwenhuijsen K, de Boer AGEM, Verbeek JHAM, et al. The Depression Anxiety Stress
Scales (DASS): detecting anxiety disorder and depression in employees absent from work due to mental health problems. Occup Environ Med 2003:60:i2 1-5.

10 Andrea H, Kant IJ, Beurskens AJHM, et al. Associations between fatigue attributions and fatigue, health, and psychosocial work characteristics: a study among employees visiting a physician in relation with fatigue. Occup Environ Med 2003;60:i99-104.

11 De Vente W, Olff M, Van Amsterdam JGC, et al. Physiological differences between burnout patients and healthy controls: blood pressure, heart rate, and cortisol responses. Occup Environ Med 2003;60:154-61.

12 Kant IJ, Bültmann U, Schroer KAP, et al. An epidemiological approach to study fatigue in the working population: the Maastricht Cohort Study. Occup Environ Med 2003;60:i32-9.

13 Jansen NWH, van Amelsvoort LGPM, Kristensen TS, et al. Work schedules and fatigue: a prospective cohort study. Occup Environ Med 2003:60:i47-53.

14 van der Ploeg E, Kleber RJ. Acute and chronic stressors among ambulance personnel: predictors of health symptoms. Occup Environ Med 2003:60:i40-6.

15 Weijman I, Ros WJG, Rutten GEHM, et al. Fatigue in employees with diabetes: its relationship with work characteristics and diabetes related burden. Occup Environ Med 2003;60:i93-8.

16 Sluiter JK, de Croon EM, Meijman TF, et al. Need for recovery from work related fatigue and its role in the development and prediction of subjective health complaints. Occup Environ Med 2003:60:i62-70.

17 Janssen N, Kant IJ, Swaen GMH, et al. Fatigue as a predictor of sickness absence: results from the Maastricht cohort study on fatigue at work. Occup Environ Med 2003;60:i71-6.

18 van Amelsvoort LGPM, Kant IJ, Bültmann U, et al. Need for recovery after work and the subsequent risk on cardiovascular disease in a working population. Occup Environ Med 2003;60:i83-7.

19 Swaen GMH, van Amelsvoort LGPM, Bültmann $U$, et al. Fatigue as a risk factor for being injured in an occupational accident: results from the Maastricht Cohort Study. Occup Environ Med 2003;60:i88-92.

20 Nieuwenhuijsen K, Verbeek JHAM, Siemerink JCM, et al. Quality of rehabilitation among workers with adjustment disorders according to practice guidelines, a retrospective cohort study. Occup Environ Med 2003;60:i77-82. 